\title{
Spin-orbit coupling and zero-field splitting of the high-spin ferric enzyme-substrate complex: Protocatechuate 3,4-dioxygenase complexed with 3,4-dihydroxyphenylacetate
}

\author{
LÜ LingLing $^{1 *}$, ZHU YuanCheng ${ }^{1}$, WANG XiaoFang ${ }^{1}$, ZUO GuoFang ${ }^{1}$, GUO Feng ${ }^{1}$, \\ ZHAO SuRui ${ }^{1} \&$ WANG YongCheng ${ }^{2}$ \\ ${ }^{1}$ College of Life Science and Chemistry, Tianshui Normal University, Tianshui 741001, China; \\ ${ }^{2}$ College of Chemistry and Chemical Engineering, Northwest Normal University, Lanzhou 730070, China
}

Received February 2, 2012; accepted May 3, 2012; published online August 12, 2012

\begin{abstract}
We used density functional calculations to investigate the electronic origins of the magnetic properties of the high-spin ferric enzyme-substrate complex protocatechuate 3,4-dioxygenase (3,4-PCD). The calculated g-tensors show that ligand-to-metal charge transfer transitions are from the protocatechuate (PCA) and Tyr408 orbitals to the Fe $\mathrm{d} \pi$ orbitals, which lead to $x$ - and $y$-polarized transitions. These polarized transitions require a spin-orbit coupling (SOC) matrix element in the $z$-direction, $L_{z}\left(z=z^{\prime}\right)$, resulting in a $g_{z^{\prime}}$ value of 2.0158, significantly deviating from 2.0023. A large zero-field splitting parameter value of $+1.147 \mathrm{~cm}^{-1}$ is due to $\Delta S=-1$ spin-orbit mixing with the quartet states for the sextet ground state, accounting for around $73 \%$ of the SOC contribution. The SOC matrix elements indicate that the high-spin $\mathrm{d}^{5}$ system Fe(III), 3,4-PCD-PCA is a weak spin-crossover compound with an $\mathrm{SOC}$ of $31.56 \mathrm{~cm}^{-1}$.
\end{abstract}

3,4-PCD-PCA, $g$-tensor, zero-field splitting, spin-orbit coupling

Citation: Lü L L, Zhu Y C, Wang X F, et al. Spin-orbit coupling and zero-field splitting of the high-spin ferric enzyme-substrate complex: Protocatechuate 3,4-dioxygenase complexed with 3,4-dihydroxyphenylacetate. Chin Sci Bull, 2013, 58: 627-633, doi: 10.1007/s11434-012-5316-7

In recent years, significant progress has been made in understanding the geometric and electronic structures of active sites of mononuclear non-heme iron enzymes [1-3] and their contributions to molecular mechanisms. A good example, the non-heme oxygenase family [1-5], performs a variety of important biological functions. These widely distributed enzymes catalyze the cleavage of $\mathrm{O}_{2}$, accompanied by insertion of both oxygen atoms into the aromatic ring of the substrate, resulting in ring cleavage.

Intradiol dioxygenases belong to a large class of enzymes called catecholic dioxygenases [6]. The catecholic dioxygenases fall into two structurally and mechanistically distinct classes, "extradiol" and "intradiol". Protocatechuate 3,4-dioxygenase $(3,4-\mathrm{PCD})$ is one of the most extensively studied intradiol dioxygenases. It catalyzes the ring cleavage of protocatechuate (PCA, 3,4-dihydroxyphenylacetate) to form $\beta$-carboxy-cis, cis-muconate, incorporating both

*Corresponding author (email: lvling100@163.com) oxygen atoms from molecular oxygen. Based on different electronic descriptions of the enzyme-substrate (ES), various mechanisms [5] have been proposed for the substrate activation step of the initial $\mathrm{O}_{2}$ attack in these reactions. However, they are very slow because they are spinforbidden. Moreover, we know that the chemistry of transition metals and their compounds is strongly influenced by the availability of multiple low-lying electronic states in these species [7-9]. This phenomenon often occurs when the formal d-electron count on the metal is $2-8$. This means that reactions involving several electronic states that may also have different spins should involve spin-conserving and spin-inversion processes. These result in a complex reaction mechanism.

Therefore, describing the electronic structure of the ES is very important to better understanding the reaction mechanism. Our motivation is to help understand the role of electronic structures in mechanistic details at a molecular level. To our knowledge, there is no reported deep theoretical 
study on the electronic origins of the magnetic properties of the high-spin ferric enzyme-substrate complex of protocatechuate 3,4-dioxygenase. In this paper we compute the $g$-tensor and zero-field splitting (ZFS) using quasi-restricted theory using density functional theory (DFT) [10] implemented with the program ORCA [11]. Then we obtain spin-orbit coupling (SOC) matrix elements using the approximate one-electron spin-orbit Hamiltonian. These calculations determine the detailed electronic structure of 3,4-PCD-PCA. The molecular orbital analysis determines the origin of the large ZFS parameter, $D$.

\section{Computational details}

\subsection{Geometrical optimization}

(1) System steps. The activate-site geometric structure of 3,4-PCD-PCA was obtained from the averaged crystallographic coordinate of $P$. putida 3,4-PCD complex with PCA (PDB code 3PCA) [12]. Hydrogen atoms were placed at standard bond lengths and angles. DFT calculations were performed on 3,4-PCD-PCA with two Me-imidazoles to model His460 and His462, 4-Me-phenolate to model Tyr408, and a bidentate PCA in the fully deprotonated state, as shown in Figure 1.

(2) DFT calculations. Spin-unrestricted DFT calculations were performed using Gaussian 03 [13] to geometrically optimize the active model described above. The double-zeta $6-31+\mathrm{G}(\mathrm{d})$ basis set was used in geometric optimization with two commonly used functionals: Becke's 1988 exchange functional with the correlation function of Perdew (BP86), and Becke's three-parameter hybrid functional with the correlation function of Lee, Yang and Parr (B3LYP). Frequencies and thermodynamic parameters were calculated, and all frequencies were found to be real. The graphical output of the molecular orbitals was generated with the program Molekel [14].

\subsection{Calculations of ZFS tensor and $g$-tensor}

A sextet state with a total spin of $S=5 / 2$ is characterized by six magnetic sublevels with $M_{s}= \pm 5 / 2, \pm 3 / 2$, and $\pm 1 / 2$. While these levels are energetically degenerate within a

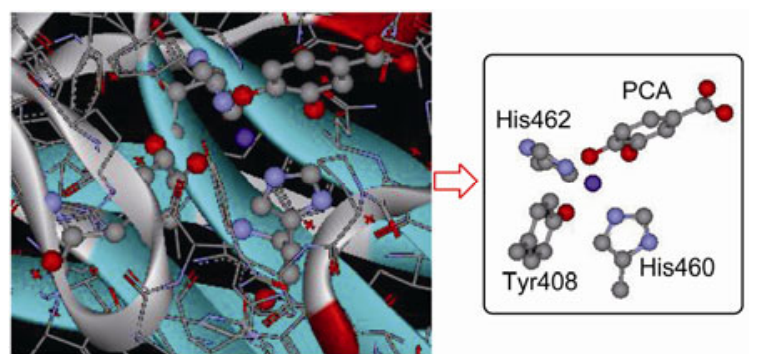

Figure 1 (Color online) Active-site geometric structures of 3,4-PCDPCA (PDB code 3PCA). nonrelativistic or scalar relativistic treatment, their degeneracy is lifted when we include SOC, dipolar spin-spin couplings and the Zeeman effect $[15,16]$. In the absence of nuclear spins and exchange interactions, the effective spin Hamiltonian of these interactions is usually written as

$$
H_{\text {spin }}=H_{\mathrm{Ze}}+H_{\mathrm{ZFS}}=\beta_{B} B g S+S D S,
$$

where $\beta_{B}$ is the Bohr magneton, $B$ is the magnetic flux density, $S$ is the effective spin operator, and $g$ and $D$ are the $g$-tensor and ZFS-tensor, respectively. $H_{\text {spin }}$ acts on the basis functions $|S M\rangle$ with $M=S, S-1, \ldots,-S$. If we choose a coordinate system that diagonalizes $D, H_{\mathrm{ZFS}}$ can be rewritten:

$$
\begin{gathered}
H_{\mathrm{ZFS}}=D\left[S_{z}{ }^{2}-1 / 3 S(S+1)\right]+E\left(S_{x}^{2}-S_{y}^{2}\right), \\
D=D_{z z}-1 / 2\left(D_{x x}+D_{y y}\right) ; \mathrm{E}=1 / 2\left(D_{x x}-D_{y y}\right) .
\end{gathered}
$$

Thus, the ZFS is uniquely defined by the parameters $D$ and $E$ and the tensor orientation. Typically, $D$ and $E / D$ are given in a coordinate system that fulfils the condition:

$$
0 \leqslant E / D \leqslant \frac{1}{3} \text {. }
$$

DFT calculations of the ZFS were carried out using qausi-restricted theory [10] by the ORCA program [11]. Previous studies $[17,18]$ have investigated the dependences of the $g$-tensor and ZFS on the exchange-correlation functional. In the case of organic radicals, various generalized gradient approximation (GGA) functionals provide nearly identical $g$-tensors and only modest but non-negligible improvement over the local density approximation (LDA) functionals. Thus, in this work, we obtain all $g$-tensor and zero-field parameters ( $D$ and $E$ ) in from additional single-point calculations using $6-31+\mathrm{G}(\mathrm{d})$ basis sets and the BP86 GGA functional. We use a recently described SOC operator that efficiently implements a second-order memory function [19].

\subsection{SOC calculations}

It is well known that, to second order in perturbation theory, the ZFS has two contributions: (a) the direct dipolar spin-spin interaction between electron pairs and (b) the SOC. Thus, to obtain more detailed SOC matrix elements, we calculate the SOC of different spin states (sextet and quartet) for the 3,4-PCD-PCA using the approximate one-electron spin-orbit Hamiltonian [20,21]:

$$
\begin{aligned}
H_{\mathrm{SO}} & =\frac{\alpha^{2}}{2} \sum_{i} \sum_{k}\left(\frac{Z_{k}^{*}}{r_{i k}^{3}}\right)\left(S_{i} \cdot L_{i k}\right)=\sum_{i} h_{i}\left(Z^{*}\right), \\
\frac{\alpha^{2}}{2} & =\frac{e^{2} h}{4 \pi m_{e}^{2} c^{2}},
\end{aligned}
$$

in which the neglect of the two-electron terms is compensated by introducing a semi-empirical parameter, here the effective nuclear charge $Z_{k}^{*} . L_{i k}$ and $S_{i}$ are the orbital and spin angular momentum operators, respectively, for electron $i$ in the framework of the nuclei indexed $k$ and $Z_{k}^{*}$ is the 
effective nuclear charge.

The root-mean-squared value of the SOC constant is defined as

$$
\langle\mathrm{SOC}\rangle=\left(\sum_{M_{S}= \pm \frac{5}{2}, \pm \frac{3}{2}, \pm \frac{1}{2}} \sum_{k=x, y, z}\left\langle{ }^{6} \psi\left(M_{S_{1}}\right)\left|H_{\mathrm{SO}}\right|{ }^{4} \psi\left(M_{S_{2}}\right)\right\rangle\right)^{1 / 2} \text {. }
$$

The SOC matrix elements in eq. (4) are evaluated by the sextet/quartet-state-averaged complete active-space selfconsistent field wave functions (CASSCF) with 5 electrons in 6 orbitals using the SOC configuration-interaction method. To qualitatively interpret nonzero SOC interaction, we also use restricted open-shell Hartree-Fock (ROHF) orbitals [22] generated at the sextet ground state to construct complete active space configuration interaction (CASCI) wave functions. All SOC calculations were performed with the GAMESS program package [23].

\section{Results and discussion}

\subsection{Geometric optimization of the 3,4-PCD-PCA complex}

The sextet state geometric model of 3,4-PCD-PCA was optimized with B3LYP using $6-31+\mathrm{G}(\mathrm{d})$ as the basis set. Figure 2 summarizes the optimization parameters. This calculation identifies the orientation of the molecular axes, in which the $x$ - and $y$-axis are in the plane of the Tyr408 ring and $z$ is out of the Tyr408 plane axis.

\subsection{Direction and orbital origins of $g$-tensor and ZFS}

(1) $g$-Tensor. The electron paramagnetic resonance $g$-tensor is a property that can be well calculated using the ORCA program by solving the coupled-perturbed SCF equations. This provides both an absolute $g$-values and $g$-shift, $\Delta g$, which represents the deviation from the free electron value,

$$
g=g_{e} \mathbf{I}+\Delta g
$$

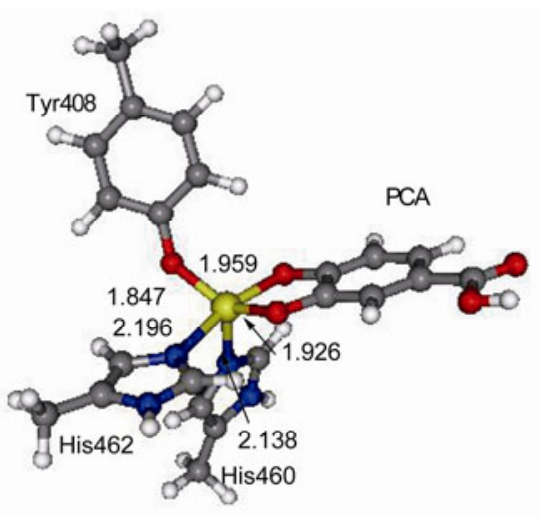

where $\mathbf{I}$ is a $3 \times 3$ unit matrix and $g_{e}=2.0023$, and

$$
\Delta g=\Delta g^{\mathrm{RMC}}+\Delta g^{\mathrm{DSO}}+\Delta g^{\mathrm{PSO}},
$$

where $\Delta g^{\mathrm{RMC}}$ is the relativistic mass correction, $\Delta g^{\mathrm{DSO}}$ is the diamagnetic spin-orbit term and $\Delta g^{\mathrm{PSO}}$ is the paramagnetic spin-orbit term [24]. The "paramagnetic" term, $\Delta g^{\mathrm{PSO}}$, dominates (except for the smallest $\Delta g$-values). Then, the Cartesian components of $\Delta g^{\mathrm{PSO}}$ may be expressed solely in terms of the occupied and virtual Kohn-Sham orbitals $\psi_{i}$ and their energies $E_{i}$ (in a.u.) [25]:

$$
\begin{aligned}
\Delta g_{\mu v}^{\mathrm{PSO}}= & \frac{\alpha^{2}}{2} g_{e}\left[\sum_{k}^{\operatorname{occ}(\alpha)} \sum_{a}^{\operatorname{virt}(\alpha)} \frac{\left\langle\psi_{k}^{\alpha}\left|H_{\mathrm{SO}, v}\right| \psi_{a}^{\alpha}\right\rangle\left\langle\psi_{a}^{\alpha}\left|l_{\mathrm{O}, u}\right| \psi_{k}^{\alpha}\right\rangle}{E_{k}^{\alpha}-E_{a}^{\alpha}}\right. \\
& \left.-\sum_{k}^{\operatorname{occ}(\beta)} \sum_{a}^{\operatorname{virt}(\beta)} \frac{\left\langle\psi_{k}^{\beta}\left|H_{\mathrm{SO}, v}\right| \psi_{a}^{\beta}\right\rangle\left\langle\psi_{a}^{\beta}\left|l_{\mathrm{O}, u}\right| \psi_{k}^{\beta}\right\rangle}{E_{k}^{\beta}-E_{a}^{\beta}}\right],
\end{aligned}
$$

here, $u$ and $v$ denote Cartesian tensor components, $\alpha$ is the fine structure constant, $H_{\mathrm{SO}}$ is the spatial part of the spin-independent spin-orbit operator, and $l_{\mathrm{O}}$ represents the orbital Zeeman operator.

As can be seen in Table 1, the $g$-tensor is found to have one distinct $\left(g_{x^{\prime}}=2.0128\right)$ and two similar values $\left(g_{y^{\prime}}=\right.$ 2.0153 and $\left.g_{z^{\prime}}=2.0158\right)$. Deviations from the free-electron $g$-value $\left(g_{e}=2.0023\right)$ are due to $\Delta S=0$ spin-orbit coupling of the excited to the ground state. The $g$-shifts are dominated by the $\Delta g^{\mathrm{PSO}}$ contributions. Our calculations show that the $\Delta g^{\mathrm{DSO}}$ and $\Delta g^{\mathrm{RMC}}$ terms contribute only about 0.0003 and -0.0007 , respectively, to $\Delta g$ (Table 1 ).

As the orbital Zeeman term does not depend on electronic

Table 1 Calculated $g$-values

\begin{tabular}{crrr}
\hline & \multicolumn{1}{c}{$x^{\prime}$} & \multicolumn{1}{c}{$y^{\prime}$} & \multicolumn{1}{c}{$z^{\prime}$} \\
\hline$\Delta g^{\mathrm{RMC}}$ & -0.0007293 & -0.0007293 & -0.0007293 \\
$\Delta g^{\mathrm{DSO}}$ & 0.0003009 & 0.0002976 & 0.0003016 \\
$\Delta g^{\text {PSO }}$ & 0.0109132 & 0.0134551 & 0.0139449 \\
$\Delta g$ & 0.0104849 & 0.0130234 & 0.0135172 \\
$g$ & 2.0128042 & 2.0153427 & 2.0158365 \\
\hline
\end{tabular}

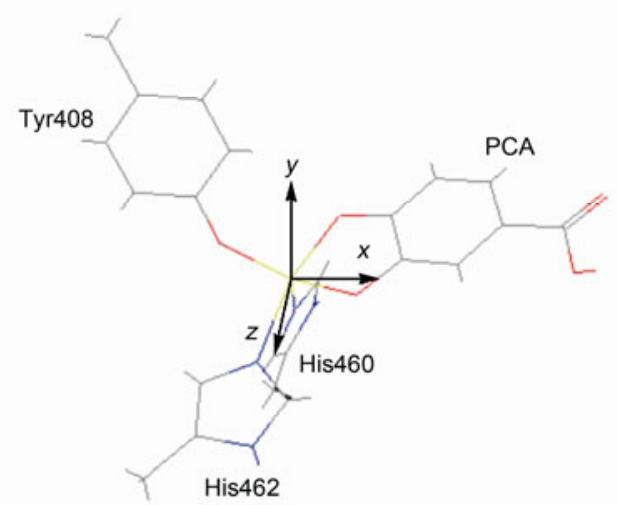

Figure 2 (Color online) Structure of 3,4-PCD-PCA showing the molecular coordinate system used in the present study. Metal ligand bond distances are given in angstroms. 
spin, the mixing of the "excitations" conserves the total spin of the system. We may thus distinguish three different types of contributions to the $\Delta g^{\mathrm{PSO}}$ term in eq. (7): $\mathbf{i}$. couplings between the (occupied) $\alpha$-spin component of the singly occupied molecular orbital (SOMO) and the $\alpha$-components of the unoccupied orbital manifold; ii. couplings between the $\beta$-components of the doubly occupied orbital manifold and the (unoccupied) $\beta$-component of the SOMO; iii. $\alpha \rightarrow \alpha$ and $\beta \rightarrow \beta$ "excitations" from doubly occupied to unoccupied orbitals.

The $\Delta S=0$ contributions from doubly occupied to unoccupied orbitals (namely, type iii) need not be considered, as any contributions from the mixing of an excited state involving a majority spin transition will be negated by a contribution from excited-state mixing of the corresponding minority spin orbital, which will have the same value but opposite signs.

We focus mainly on couplings of type ii. Contributions from transitions from doubly occupied to singly occupied orbitals result in a positive contribution to $\Delta g_{u v}$. The $g$-tensor is the property that probably provides the most compact experimental image of the spin-density distribution in a molecule. It is clear that a large spin-orbit (SO) coupling constant of the metal compared to small SO coupling with only light ligand atoms should lead to larger $g$-tensor anisotropy $\left(g^{\text {aniso }}=0.003\right.$ ) with increased spin density on the metal. Figure 3 shows the plot of a spin-density isosurface for 3,4-PCD-PCA with $\rho^{\alpha-\beta}=+0.01$. The expected large spin density on the $\mathrm{Fe}$ atom is seen, with a maximum value of $\rho^{\alpha-\beta}(\mathrm{Fe})=3.99$ a.u. As shown in our orbital analysis, all of the observed ligand-to-metal charge transfer (LMCT) transitions are from the PCA and Tyr408 orbitals to the Fe $\mathrm{d} \pi$ orbitals, which are either $x$-polarized and originating from PCA or $y$-polarized and originating from Try408. These $x$ - and $y$-polarized transitions require an SOC matrix element in the $z$-direction, $L_{z}\left(z=z^{\prime}\right)$. Moreover the SOC is effectively a localized, one-center (Fe atom), one-electron

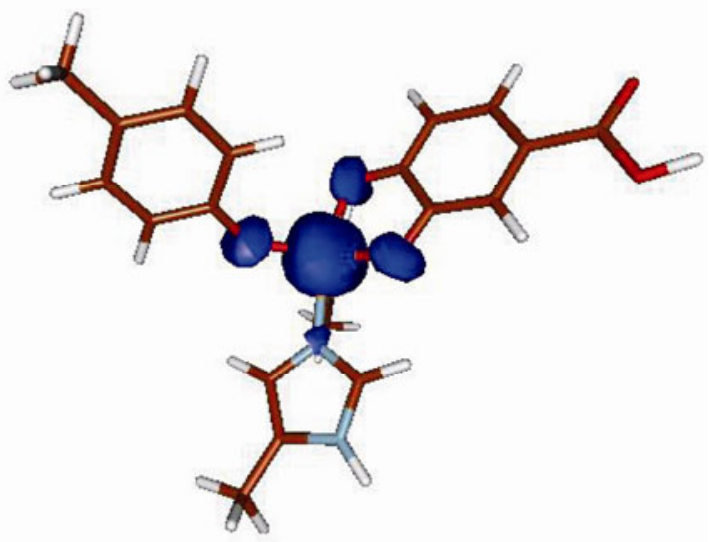

Figure 3 (Color online) Spin-density isosurface (+0.01 a.u.) for 3,4PCD-PCA. operator, which leads to the $g_{z^{\prime}}$ value 2.0158 , significantly deviating from 2.0023 in the $z^{\prime}$-direction. At the same time, a larger $g_{y^{\prime}}=2.0153$ is due to transition from the doubly occupied $\mathrm{d} x y$ orbital to the singly occupied $\mathrm{d} y z$ orbital, i.e. $L_{y^{\prime}}$ coupled $\left(x=y^{\prime}\right)$. We note that the molecular unique magnetic axis $\left(z^{\prime}\right)$ is equivalent to the $z$-axis, consistent with the calculated $g$-matrix, while the $x$ and $y$ axes are interchanged: $x=y^{\prime}$ and $y=x^{\prime}$. The magnetic axes are plotted in Figure 4.

(2) $D$-tensor. BP86 calculations were performed to determine the signs of the $D$ and $E$ values for 3,4-PCD-PCA. The $D$-tensor was mapped onto the coordinates of the 3,4-PCD-PCA active site (see Figure 4). By diagonalizing the $D$-tensor, the principal values $D_{x x^{\prime}}\left(4.601 \mathrm{~cm}^{-1}\right), D_{y y^{\prime}}$ $\left(5.361 \mathrm{~cm}^{-1}\right)$, and $D_{z z^{\prime}}\left(6.128 \mathrm{~cm}^{-1}\right)$ are determined, and the spin-Hamiltonian parameters $\left(D=D_{z z^{\prime}}-1 / 2\left(D_{x x^{\prime}}+D_{y y^{\prime}}\right), E=\right.$ $\left.1 / 2\left(D_{x x^{\prime}}-D_{y y^{\prime}}\right)\right)$ are calculated $\left(D=+1.147 \mathrm{~cm}^{-1}\right.$ and $E / D=$ +0.332 ). We find that the $D$-tensor orientations consist of $x^{\prime}$ and $y^{\prime}$-directions in the plane of the Tyr408 ring and the $z^{\prime}$-direction oriented perpendicular to the Tyr408 plane axis and closest to the $\mathrm{O}^{\mathrm{PCA}}-\mathrm{O}^{\mathrm{PCA}}$ line.

Table 2 gives the detailed results of the ZFS calculation using Quasi-restricted DFT. From the results in Table 2, the ZFS contains two significant contributions: a first-order term involving the direct dipolar spin-spin (SS) interaction between pairs of electrons and a second-order term from the SOC interaction. The major contribution arises from the second-order SOC contribution (around $98 \%$ of $D$ ), while the SS contributions are essentially negligible. The SOC part contains three significant contributions. The major contribution comes from the $\alpha \rightarrow \beta$ spin-flip excitations. This excitation contributes around $73 \%$ of the $D_{\text {SOC }}$ value, corresponding to the spin-pairing $\Delta S=-1$ (i.e. ${ }^{6} \Gamma \rightarrow{ }^{4} \Gamma$ with one unpaired electron in the excited state) transitions. The second largest SOC contribution arises from the $\beta \rightarrow \alpha$

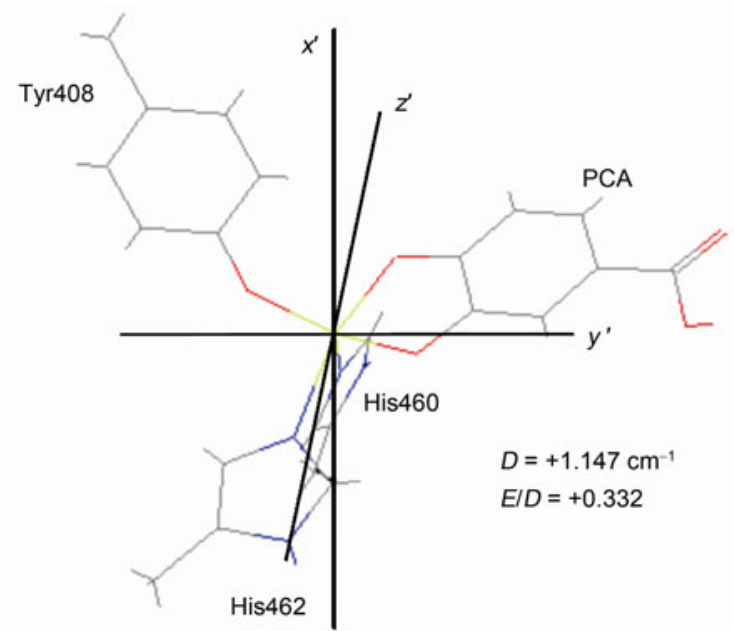

Figure 4 (Color online) Orientations of the ZFS tensors for 3,4PCD-PCA 
Table 2 Contributions to the calculated ZFS between SOC and SS (all values are in $\mathrm{cm}^{-1}$ )

\begin{tabular}{ccccc}
\hline Method & Individual contributions & & $D$ & $E$ \\
& & $\alpha \rightarrow \alpha$ & 0.021 & 0.020 \\
& & $\beta \rightarrow \beta$ & 0.115 & 0.055 \\
BP86-QR-SOMF & Spin-orbit-coupling & $\alpha \rightarrow \beta$ & 0.820 & 0.214 \\
/6-31+G(d) & (SOC) & $\beta \rightarrow \alpha$ & 0.165 & 0.087 \\
& & Total & 1.121 & 0.376 \\
\cline { 2 - 5 } & & Coulomb & 0.03118 & 0.00501 \\
& \multirow{2}{*}{ Spin-spin(SS) } & Exchange & -0.00542 & -0.00105 \\
& & Total & 0.02576 & 0.00396 \\
\hline
\end{tabular}

spin-raising $\Delta S=+1$ excitations (around $15 \%$ of $D_{\mathrm{SOC}}$ ) corresponding to LMCT transitions. The final major SOC contribution comes from the $\beta \rightarrow \beta$ excitations. These correspond primarily to the spin-allowed $\Delta S=0\left({ }^{6} \Gamma \rightarrow{ }^{6} \Gamma\right)$ ligand-field excitations and have usually been solely held responsible for the ZFS. However, compared to the $D$ value, they contribute only around $0.1 \mathrm{~cm}^{-1}(\sim 10 \%)$, which renders arguments based only on spin-allowed ligand-field excitations in high-spin ${ }^{5} \mathrm{~d}$ systems unreliable.

The three contributions are summed to give the total $D$. We find that the $\Delta S=-1$ contributions make significant contributions to $D_{x x^{\prime}}, D_{y y^{\prime}}$, and $D_{z z^{\prime}}$, with the primary contribution arising from the single-determinant spin-paired states within the single occupied orbital sets, $\varphi_{2}=$ $d_{y z}+d_{x y}$ (hybrid) $+\pi_{\mathrm{PCA}}, \varphi_{3}=d_{y z}+\pi_{\mathrm{Tyr} 408}$, and $\varphi_{4}=d_{x z}+\sigma_{\mathrm{PCA}}$ (see Figure 5). Namely, $D_{y y^{\prime}}^{(-)}\left(\varphi_{2} \uparrow \rightarrow \varphi_{4} \downarrow\right)$ and $D_{z z^{\prime}}^{(-)}\left(\varphi_{2} \uparrow \rightarrow\right.$ $\left.\varphi_{3} \downarrow\right)$ spin-flip excitations make the largest contributions to the SOC and lead to the larger values of $D_{y y^{\prime}}\left(5.361 \mathrm{~cm}^{-1}\right)$ and $D_{z z^{\prime}}\left(6.128 \mathrm{~cm}^{-1}\right)$. We note that $g_{y^{\prime}}$ and $g_{z^{\prime}}$-tensors are larger due to $\Delta S=0 \mathrm{SOC}$ into the ground state via $L_{y^{\prime}}$ and $L_{z^{\prime}}$. These calculations show that it is necessary to consider second-order $\Delta S= \pm 1$ spin-orbit mixing to account for this unusually large value for $D$. This also identifies the origin of large ZFS as spin-orbit coupling to low-lying $\Delta S=-1$ excited states, and shows that the unique magnetic axis $\left(z^{\prime}\right)$ is equivalent to the molecular $z$-axis, consistent with the computed $g$-tensor.

(3) SOC matrix. Based on the above discussion, we find that the $\Delta S=-1$ spin-flip transition states make significant contributions to $D$. However, A spin-forbidden transition requires an SOC effect that provides a major spin-flip mechanism [20,21]. The SOC induces a spin-multiplicity mixing that allows the wave function to break spin symmetry, and the magnitude of the spin-multiplicity mixing increases in a small energy gap between high- and low-spin states. Therefore, we must inspect the orbital relationships that promote the SOC matrix elements.

We next turn our attention to the SOC computation. Using a sextet calculation, the ROHF orbitals for constructing the quartet and sextet CASCI wave functions to be used in the SOC evaluation have been generated in 3,4-PCD-PCA. At least five active orbitals, as depicted in

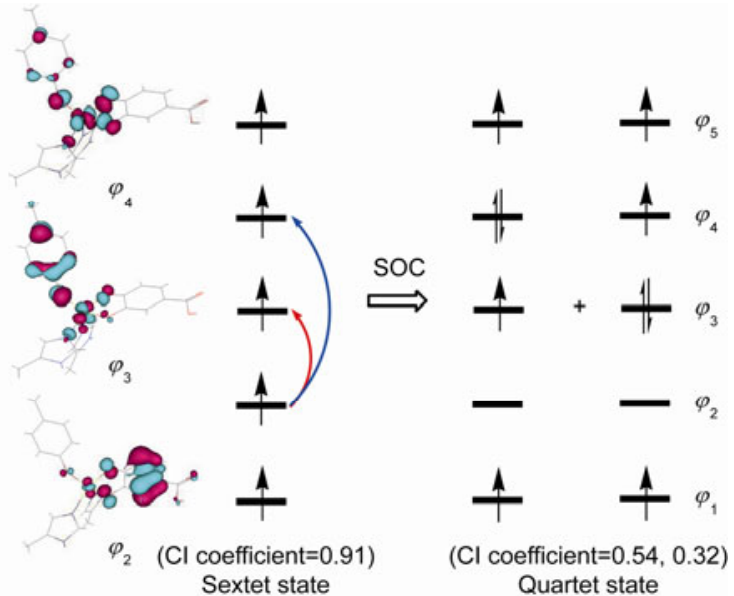

Figure 5 (Color online) Schematic of the required electronic structure change for the $\Delta S=-1$ spin-orbit mixing (sextet $\rightarrow$ quartet transformation). The double-occupied non-active orbitals are omitted to save space.

Figure 5 (with non-vital SOC orbitals, $\varphi_{1}$ and $\varphi_{5}$ omitted to save space). Interestingly, the 3,4-PCD-PCA complex shows significant multireference character for the quartet state. At the CAS $(5,6)$ level, the dominant determinants of the MCSCF wave functions have configuration interaction (CI) coefficients of 0.91 and 0.06 for the sextet state and $0.54,0.32,0.13$, and -0.18 for the quartet state (configurations of less than 0.1 have not been listed). Thus the permissible approximation of the sextet wave function by a single configuration (0.91) enables us to analyze the SOC matrix elements $(0.13$ and $-0.18 \mathrm{CI}$ are not considered due to the smaller CI coefficients). Thus we see that the quartet configuration is described by the superposition of two vital "closed-shell" configurations,

$$
\left|{ }^{4} \psi\right\rangle=0.54\left|{ }^{4} \phi_{1} \phi_{3} \phi_{5}\right\rangle+0.32\left|{ }^{4} \phi_{1} \phi_{4} \phi_{5}\right\rangle,
$$

where $i={ }^{4} \varphi_{1} \varphi_{3} \varphi_{5}$ and ${ }^{4} \varphi_{1} \varphi_{4} \varphi_{5}$ (see Figure 5). Nonzero elements of the $k$-components $(k=x, y$, and $z)$ of the SOC matrix, $\left\langle{ }^{6} \psi\left(M_{S_{1}}\right)\left|H_{\text {so }}\right|{ }^{4} \psi\left(M_{S_{2}}\right)\right\rangle_{k}$, are always proportional to the function, $F_{k}$, given by

$$
F_{k}=2^{-1 / 2} \times 0.54\left\langle\phi_{2}\left|H_{\mathrm{SO}, k}\right| \phi_{4}\right\rangle+2^{-1 / 2} \times 0.32\left\langle\phi_{2}\left|H_{\mathrm{SO}, k}\right| \phi_{3}\right\rangle \text {. }
$$

To further understand the efficient SOC, it is very important to discuss the SOC matrix elements $\left\langle\phi_{2}\left|H_{\mathrm{SO}, k}\right| \phi_{4}\right\rangle$ 
and $\left\langle\phi_{2}\left|H_{\mathrm{SO}, k}\right| \phi_{3}\right\rangle$. Because the SOC constant $\left(\zeta_{\mathrm{Fe}}\right)$ is an order of magnitude greater than the SOC values for oxygen and carbon, it is a reasonable approximation to consider only the Fe contribution when discussing spin-orbit mixing with quartet states. Thus

$$
\begin{aligned}
\langle\mathrm{SOC}\rangle & =\eta C_{0} C_{j 1} \sum_{k} \xi_{\mathrm{Fe}}\left\langle\phi_{2}\left|L_{\mathrm{Fe}, k}\right| \phi_{4}\right\rangle\left\langle\theta_{2}\left|S_{k}\right| \theta_{4}\right\rangle \\
& +\eta C_{0} C_{j 2} \sum_{k} \xi_{\mathrm{Fe}}\left\langle\phi_{2}\left|L_{\mathrm{Fe}, k}\right| \phi_{3}\right\rangle\left\langle\theta_{2}\left|S_{k}\right| \theta_{3}\right\rangle,
\end{aligned}
$$

where $\eta$ is the $M_{S}$-dependent weighing factor, and $\theta=\alpha$ and/or $\beta$. In this case, for the sextet state, the fundamental open-shell configuration has one dominant coefficient, i.e. $C_{0}=0.91 \approx 1$, while the coefficients for quartet states are $C_{j 1}$ $=0.54$ and $C_{j 2}=0.32$. For $\left\langle S_{1}=5 / 2 ; M s_{1 k}\left|H_{\mathrm{SO}, k}\right| S_{2}=\right.$ $\left.3 / 2 ; M s_{2 k}\right\rangle$ matrix elements, the calculated SOC matrix elements come mainly from the $\varphi_{2} \rightarrow \varphi_{4}$ and $\varphi_{2} \rightarrow \varphi_{3}$ spin flip. Therefore:

$$
\begin{aligned}
\left\langle\phi_{2}\left|L_{\mathrm{Fe}, k}\right| \phi_{3}\right\rangle\left\langle\theta_{2}\left|S_{k}\right| \theta_{3}\right\rangle= & \left\langle d_{y z}+d_{x y}\left|L_{\mathrm{Fe}, x}\right| d_{y z}\right\rangle\left\langle\alpha\left|S_{x}\right| \beta\right\rangle \\
& +\left\langle d_{y z}+d_{x y}\left|L_{\mathrm{Fe}, y}\right| d_{y z}\right\rangle\left\langle\alpha\left|S_{y}\right| \beta\right\rangle \\
& +\left\langle d_{y z}+d_{x y}\left|L_{\mathrm{Fe}, z}\right| d_{y z}\right\rangle\left\langle\alpha\left|S_{z}\right| \alpha\right\rangle .
\end{aligned}
$$

Here, only the second term is non-zero. This shift creates an angular momentum along an axis perpendicular to the plane spanned by the atomic orbitals. An illustration is given in Figure 5, where the two molecular orbitals (MOs) are $\varphi_{2}$ and $\varphi_{3}$, and the electron shift from $\varphi_{2}\left(d_{y z}+d_{x y}\right)$ to $\varphi_{4}\left(d_{x z}\right)$ creates an angular momentum in the $L_{y}$-direction $\left(y=x^{\prime}\right)$, resulting in the ZFS of $D_{x x^{\prime}}=4.601 \mathrm{~cm}^{-1}$ and the smaller SOC matrix elements in the $y^{\prime}$ direction (see Table 3). Similarly,

$$
\begin{aligned}
\left\langle\phi_{2}\left|L_{\mathrm{Fe}, k}\right| \phi_{4}\right\rangle\left\langle\theta_{2}\left|S_{k}\right| \theta_{4}\right\rangle= & \left\langle d_{y z}+d_{x y}\left|L_{\mathrm{Fe}, x}\right| d_{x z}\right\rangle\left\langle\alpha\left|S_{x}\right| \beta\right\rangle \\
& +\left\langle d_{y z}+d_{x y}\left|L_{\mathrm{Fe}, y}\right| d_{x z}\right\rangle\left\langle\alpha\left|S_{y}\right| \beta\right\rangle \\
& +\left\langle d_{y z}+d_{x y}\left|L_{\mathrm{Fe}, z}\right| d_{x z}\right\rangle\left\langle\beta\left|S_{z}\right| \beta\right\rangle .
\end{aligned}
$$

In eq. (12), the second term is zero due to the mismatch for the $d_{y z}+d_{x y} \rightarrow d_{x z}$ transition in the $L_{y}$ angular momentum direction. The first and third terms produce the angular momentum in the $L_{x}\left(x=y^{\prime}\right)$ and $L_{z}\left(z=z^{\prime}\right)$ directions, respectively, and value in the $L_{z}\left(z=z^{\prime}\right)$ direction is larger for the dominant mixed weights of $d_{y z}$, leading to the larger ZFS $D_{z z^{\prime}}$ of $6.128 \mathrm{~cm}^{-1}$. The one-electron SOC value calculated is $31.56 \mathrm{~cm}^{-1}$ in the $3,4-\mathrm{PCD}-\mathrm{PCA}$ complex. It is noteworthy that the SOC values are also related to the MO's delocalization, the direction of the orbital's rotation, and the configuration coefficient $\left(C_{j}\right)$ of the quartet states $[9,26]$. These analysis results are consistent with the calculated SOC (see Table 3 ) and $D$-tensors (see Table 2). Further, these also indicate that the significant contributions to $D$ arise from the $\Delta S=-1$ spin-flip transition, and that the highspin ground state, 3,4-PCD-PCA, is a weak spin-crossover compound ( $\mathrm{SOC}=31.56 \mathrm{~cm}^{-1}$ ) compared with the Fe SOC constant $\left(\zeta_{\mathrm{Fe}}=407.23 \mathrm{~cm}^{-1}\right)$.

\section{Conclusions}

We have studied the electronic origins of the magnetic properties of 3,4-PCD-PCA using theoretical calculations. The geometric model of the high-spin 3,4-PCD-PCA compound have been determined and characterized at the DFT-B3LYP/6-31+G(d) level. We carried out BP86 calculations of the ZFS and $g$-tensors using quasi-restricted theory, which was implemented in the program ORCA. The SOC between the quartet and sextet states was calculated using the SO-CI method with the converging CASSCF wave function using the GAMESS program package. From the calculations the following conclusions emerged:

(1) The calculated $g$-tensors show that LMCT transitions are from the PCA and Tyr408 orbitals to the Fe $\mathrm{d} \pi$ orbitals, which will lead to the $x$ - and $y$-polarized transitions. These polarized transitions require an SOC matrix element in the

Table 3 Calculated SOC matrix elements $\left(\mathrm{cm}^{-1}\right)$ in the 3,4-PCD-PCA structure

\begin{tabular}{ccc}
\hline State $1 \quad\left\langle H_{\mathrm{SO}}\right\rangle_{r}$ & $\left\langle S_{1}=5 / 2 ; M s_{1 k}\left|H_{\mathrm{SO}, k}\right| S_{2}=3 / 2 ; M s_{2 k}\right\rangle$ & SOC \\
\hline & $\left\langle M s_{1}=3 / 2\left|H_{\mathrm{SO}}\right| M s_{2}=3 / 2\right\rangle=7.29$ \\
& $\left\langle M s_{1}=1 / 2\left|H_{\mathrm{SO}}\right| M s_{2}=1 / 2\right\rangle=8.93$ \\
& $\left\langle M s_{1}=-1 / 2\left|H_{\mathrm{SO}}\right| M s_{2}=-1 / 2\right\rangle=8.93$ \\
& $\left\langle M s_{1}=-3 / 2\left|H_{\mathrm{SO}}\right| M s_{2}=-3 / 2\right\rangle=7.29$ \\
\hline${ }^{6} \Gamma$ & $\left\langle M s_{1}=5 / 2\left|H_{\mathrm{SO}}\right| M s_{2}=3 / 2\right\rangle=-11.94 ;-6.34$ \\
& $\left\langle M s_{1}=-5 / 2\left|H_{\mathrm{SO}}\right| M s_{2}=-3 / 2\right\rangle=-11.94 ; 6.34$ \\
& $\left\langle M s_{1}=3 / 2\left|H_{\mathrm{SO}}\right| M s_{2}=1 / 2\right\rangle=-9.24 ;-4.91$ \\
& $\left\langle M s_{1}=-3 / 2\left|H_{\mathrm{SO}}\right| M s_{2}=-1 / 2\right\rangle=-9.24 ; 4.91$ \\
& $\left\langle M s_{1}=1 / 2\left|H_{\mathrm{SO}}\right| M s_{2}=-1 / 2\right\rangle=-6.54 ;-3.47$ \\
& $\left\langle M s_{1}=-1 / 2\left|H_{\mathrm{SO}}\right| M s_{2}=1 / 2\right\rangle=-6.54 ; 3.47$ \\
& $\left\langle M s_{1}=-1 / 2\left|H_{\mathrm{SO}}\right| M s_{2}=-3 / 2\right\rangle=-3.77 ;-2.01$ \\
& $\left\langle M s_{1}=1 / 2\left|H_{\mathrm{SO}}\right| M s_{2}=3 / 2\right\rangle=-3.77 ; 2.01$ \\
\hline
\end{tabular}


$z$-direction, $L_{z}\left(z=z^{\prime}\right)$. Moreover the SOC is effectively a localized, one-center (Fe atom), one-electron operator, resulting in the $g_{z^{\prime}}$ value 2.0158 , significantly deviating from 2.0023 in the $z^{\prime}$-direction.

(2) We performed BP86 calculations to determine the signs and values of the $D$ and $E$ parameters for $3,4-\mathrm{PCD}-\mathrm{PCA}$, and found that $D=+1.147 \mathrm{~cm}^{-1}$ and $E / D=$ +0.332 . We found that the $\Delta S=-1$ contributions to $D_{x x^{\prime}}, D_{y y^{\prime}}$, and $D_{z z^{\prime}}$ were significant, around $73 \%$ of the $D_{\text {SOC }}$ value. The primary contribution arises from the $D_{y y^{\prime}}^{(-)}\left(\varphi_{2} \uparrow \rightarrow \varphi_{4} \downarrow\right)$ and $D_{z z^{\prime}}^{(-)}\left(\varphi_{2} \uparrow \rightarrow \varphi_{3} \downarrow\right)$ spin-flip excitation.

(3) A spin-forbidden transition requires an SOC effect that provides a major spin-flip mechanism, but the calculated SOC shows that the high-spin ground state, 3,4-PCD-PCA, is a weak spin-crossover compound with an SOC of $31.56 \mathrm{~cm}^{-1}$.

This work was supported by the "QingLan" Talent Engineering Funds through TianShui Normal University and the Key Project of the Chinese Ministry of Education (211189).

1 Costas M, Mehn M P, Jensen M P, et al. Dioxygen activation at mononuclear nonheme iron active sites: Enzymes, models, and intermediates. Chem Rev, 2004, 104: 939-986

2 Solomon E I, Brunold T C, Davis M I, et al. Geometric and electronic structure/function correlations in non-heme iron enzymes. Chem Rev, 2000, 100: 235-349

3 Nam W. High-valent iron(IV)-oxo complexes of heme and non-heme ligands in oxygenation reactions. Acc Chem Res, 2007, 40: 522-531

4 Solomon E I. Geometric and electronic structure contributions to function in bioinorganic chemistry: Active sites in non-heme iron enzymes. Inorg Chem, 2001, 40: 3656-3669

5 Pau M Y M, Davis M I, Orville A M, et al. Spectroscopic and electronic structure study of the enzyme-substrate complex of intradiol dioxygenases: Substrate activation by a high-spin ferric non-heme iron site. J Am Chem Soc, 2007, 129: 1944-1958

6 Lipscomb J D, Orville A M. Degradation of Environmental Pollutants by Microorganisms and Their Metalloenzymes. New York: Marcel Dekker, Inc., 1992. 243-298

7 Fiedler A, Schroder D, Shaik S, et al. Electronic structures and gas phase reactivities of cationic late-transition-metal oxides. J Am Chem Soc, 1994, 116: 10734-10741

8 Yoshizawa K, Shiota Y, Yamabe T. Intrinsic reaction coordinate analysis of the conversion of methane to methanol by an iron-oxo species: A study of crossing seams of potential energy surfaces. J Chem Phys, 1999, 111: 538-545

9 Lü L L, Wang Y C, Wang Q, et al. Why is $\mathrm{Pt}_{4}{ }^{+}$the least efficient cationic cluster in activating the $\mathrm{C}-\mathrm{H}$ bond in methane? Two-state reac- tion computational investigation. J Phys Chem C, 2010, 114: 17610-17620

10 Neese F. Importance of direct spin-spin coupling and spin-flip excitations for the zero-field splittings of transition metal complexes: A case study. J Am Chem Soc, 2006, 128: 10213-10222

11 Neese F. ORCA-An $a b$ initio, density functional and semiempirical program package. Version 2.8-20. Mülheim an der Ruhr: MaxPlanck Institute for Bioinorganic Chemistry, 2010

12 Elgren T E, Orville A M, Kelly K A, et al. Crystal structure and resonance Raman studies of protocatechuate 3,4-dioxygenase complexed with 3,4-dihydroxyphenylacetate. Biochemistry, 1997, 36: 11504-11513

13 Frisch M J. Gaussian 03 (Revision-E.01). Gaussian Inc., Pittsburgh PA, 2003

14 Flükiger P, Lüthi H P, Portmann S, et al. Molekel 4.0. Manno: Swiss Center for Scientific Computing, 2000

15 Sinnecker S, Neese F. Spin-spin contributions to the zero-field splitting tensor in organic triplets, carbenes and biradicals-A density functional and $a b$ initio study. J Phys Chem A, 2006, 110: 12267-12275

16 Neese F, Edward I, Solomon E I. Calculation of zero-field splittings, $g$-values, and the relativistic nephelauxetic effect in transition metal complexes. Application to high-spin ferric complexes. Inorg Chem, 1998, 37: 6568-6582

17 Skulan A J, Hanson M A, Hsu H F, et al. EPR spectroscopy of $\left[\mathrm{Fe}_{2} \mathrm{O}_{2}\left(5-\mathrm{Et}_{3}-\mathrm{TPA}\right)_{2}\right]^{3+}$ : Electronic origin of the unique spinhamiltonian parameters of the $\mathrm{Fe}_{2}{ }^{\mathrm{IIIIV}} \mathrm{O}_{2}$ diamond core. Inorg Chem, 2003, 42: 6489-6496

18 Neese F. Calculation of the zero-field splitting tensor on the basis of hybrid density functional and Hartree-Fock theory. J Chem Phys, 2007, 127: 164112-164119

19 Hess B A, Marian C M, Wahlgren U, et al. A mean-field spin-orbit method applicable to correlated wavefunctions. Chem Phys Lett, 1996, 251: 365-371

20 Danovich D, Marian C M, Neuheuser T, et al. Spin-orbit coupling patterns induced by twist and pyramidalization modes in $\mathrm{C}_{2} \mathrm{H}_{4}$ : A quantitative study and a qualitative analysis. J Phys Chem A, 1998, 102: $5923-5936$

21 Koseki S, Fedorov D G, Schmidt M W, et al. Spin-orbit splittings in the third-row transition elements: Comparison of effective nuclear charge and full Breit-Pauli calculations. J Phys Chem A, 2001, 105: 8262-8268

22 Isobe H, Yamanaka S, Kuramitsu S, et al. Regulation mechanism of spin-orbit coupling in charge-transfer-induced luminescence of imidazopyrazinone derivatives. J Am Chem Soc, 2008, 130: 132-149

23 Granovsky A A. GAMESS program. Moscow: Moscow State University

24 Kaupp M, Asher J, Arbuznikov A, et al. Understanding the unusual $g$-values and the spin density distribution of hydrogen atoms trapped in silasesquioxanesy. Phys Chem Chem Phys, 2002, 4: 5458-5466

25 Remenyi C, Kaupp M. Where is the spin? Understanding electronic structure and $g$-tensors for ruthenium complexes with redox-active quinonoid ligands. J Am Chem Soc, 2005, 127: 11399-11413

26 Lü L L, Wang Y C, Liu H W, et al. Theoretical study of spin-orbit coupling and kinetics in spin-forbidden reaction between $\mathrm{Ta}\left(\mathrm{NH}_{2}\right)_{3}$ and $\mathrm{N}_{2} \mathrm{O}$. Theor Chem Acc, 2010, 127: 507-517

Open Access This article is distributed under the terms of the Creative Commons Attribution License which permits any use, distribution, and reproduction in any medium, provided the original author(s) and source are credited. 